\title{
OCORRÊNCIA DE Erythmelus tingitiphagus (HYMENOPTERA: MYMARIDAE) NA BORDADURA DE TALHÕES DE SERINGUEIRA VIZINHOS AO CERRADO E MATA RIPÁRIA EM ITIQUIRA, MT, BRASIL
}

\author{
Rodrigo Souza Santos ${ }^{1}$, Joaquim Manoel da Silva ${ }^{2}$, Romeu de Carvalho Andrade Neto ${ }^{1}$ \\ ${ }^{1}$ Embrapa Acre - Rod. BR-364, Km 14, CP. 321, CEP: 68908-970, Rio Branco, AC, Brasil. E-mail: rodrigo@cpafac.embrapa.br; \\ romeu@cpafac.embrapa.br \\ ${ }^{2}$ Universidade do Estado do Mato Grosso (UNEMAT) - Rod. BR-158, Km 148, CP. 08, CEP: 78690-000, Nova Xavantina, MT, \\ Brasil. E-mail: joaquim.manoel@gmail.com
}

\section{RESUMO}

O efeito de borda e a preservação da vegetação de entorno ou vegetação nativa próximas aos monocultivos atuam diretamente na manutenção dos inimigos naturais na área, promovendo aumento ou diminuição populacional destes agentes de controle biológico. Neste sentido, o objetivo do trabalho foi verificar a ocorrência do parasitoide de ovos Erythmelus tingitiphagus, em árvores da bordadura em dois talhões comerciais de seringueiras do clone PR 255 vizinhos ao Cerrado e Mata Ripária na fazenda da empresa "Plantações Edouard Michelin Ltda.", Itiquira, MT. Quinzenalmente oito folhas maduras foram coletadas em cinco árvores da bordadura de cada um dos talhões, processadas em laboratório e analisadas sob estereoscópio após cinco dias. $\mathrm{O}$ número de parasitoides adultos foi contabilizado e a associação entre o parasitoide e a vegetação adjacente foi verificada pelo teste do Qui-quadrado $(\alpha=5 \%)$, a abundância populacional pelo teste de $\mathrm{T}$ de Student $(\alpha=1 \%)$ e a uniformidade ao longo das linhas pelo teste de Kruskal-Wallis $(\alpha=1 \%)$. A maior ocorrência de E. tingitiphagus foi verificada no talhão vizinho ao Cerrado, além de um efeito negativo do efeito de borda sobre a população do parasitoide.

Palavras-chave: Chalcidoidea, efeito de borda, Hevea brasiliensis, Leptopharsa heveae

\section{OCCURRENCE OF Erythmelus tingitiphagus (HYMENOPTERA: MYMARIDAE) IN THE BORDER OF RUBBER TREE PLOTS NEXT TO SAVANNA AND RIPARIAN FOREST IN ITIQUIRA, MT, BRAZIL}

\begin{abstract}
The edge effect and the preservation of the neighboring vegetation or native vegetation next to monocultives act directly on the maintenance of natural enemies in the area, promoting a population increase or decrease of these biological control agents. In this way, the aim of this study was to verify the occurrence of the lace bug egg parasitoid Erythmelus tingitiphagus in the border trees of two plots of rubber tree clone PR 255 close to Savanna and Riparian forest in the farm of "Plantações Edouard Michelin Ltda."company, county of Itiquira, MT, Brazil. Every 15 day eight mature leaves were collected from five trees in the border of the plots, processed in laboratory and analyzed under stereoscope after five days. The number of adult parasitoids was counted and the association between the parasitoid and adjacent vegetation was verified by Chisquare test $(\alpha=5 \%)$, the population abundance by T Student's test $(\alpha=1 \%)$ and uniformity along the lines using the Kruskal-Wallis test $(\alpha=1 \%)$. The highest occurrence of E. tingitiphagus was verified in the plot near the Cerrado, in addition to a negative effect of edge effect in the parasitoid population.
\end{abstract}

Key words: Chalcidoidea, edge effect, Hevea brasiliensis, Leptopharsa heveae 


\section{INTRODUÇÃO}

A transição abrupta entre dois ambientes adjacentes por uma mudança, biótica e/ou abiótica pode produzir mudanças na zona de transição, tais como alterações na composição de espécies e microclimas, podendo alterar, de forma acentuada, os processos ecológicos que ocorrem no fragmento, constituindo o chamado "efeitos de borda" (Murcia, 1995), que pode controlar o fluxo de organismos entre fragmentos florestais (Cadenasso \& Pickett, 2000).

Segundo Altieri (1994) as bordas são importantes para a propagação e proteção de uma ampla gama de agentes naturais de controle biológico de pragas agrícolas, promovendo a movimentação de artrópodes benéficos, das margens da vegetação de entorno para dentro das plantações (Altieri et al. 2003). Entretanto, alguns autores afirmam que a formação de bordas pode alterar a distribuição, o comportamento e a sobrevivência de espécies, tanto de plantas como de animais, podendo causar extinções de espécies e, conseqüentemente, de suas interações (Murcia, 1995; Girão et al., 2007; Silva et al., 2007).

Assim, a comunidade biológica pode ter diferentes respostas aos efeitos de borda, sendo que são favorecidas as espécies que conseguem se adaptar às condições microclimáticas deste habitat. Dentre estas espécies sujeitas ao efeito de borda, destacam-se os himenópteros parasitoides, abundantes na natureza e ocupam os mais diversos tipos de ambientes disponíveis em praticamente todos os ecossistemas terrestres, sendo importantes reguladores naturais de populações de insetos-praga (Borror \& DeLong 1988; LaSalle \& Gauld 1993; Marchiori et al., 2001; Gallo et al., 2002).

A preservação da vegetação de entorno ou vegetação nativa próximas aos monocultivos propicia condições favoráveis (fontes alimentares, hospedeiros alternativos e áreas de refúgio) à manutenção dos parasitoides e outros inimigos naturais na área (Altieri, 1994). Neste sentido, o objetivo do trabalho foi verificar a ocorrência de Erythmelus tingitiphagus (Soares) (Hymenoptera: Mymaridae), parasitoide de ovos do percevejo-de-renda da seringueira Leptopharsa heveae Drake \& Poor (Hemiptera: Tingidae) (Costa et al. 2003; Santos \& Freitas, 2008), em árvores da bordadura de dois talhões de seringueira, vizinhos a um fragmento de Cerrado e Mata ripária, sob influência do efeito de borda.

\section{MATERIAL E MÉTODOS}

O trabalho foi desenvolvido em dois talhões comerciais de seringueira do clone PR 255 vizinhos a um fragmento de Cerrado e Mata ripária na fazenda da empresa "Plantações Edouard Michelin Ltda."

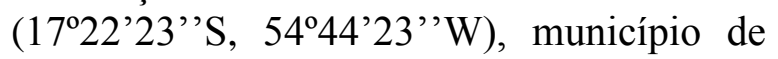
Itiquira, MT (Tabela 1). As árvores foram cultivadas em regime tradicional de monocultivo, com espaçamento de $2,5 \mathrm{~m}$ x 8 $\mathrm{m}$ e receberam tratamento convencional com produtos fitossanitários intercalados (Methomyl $^{\circledR}, \quad$ Monocrotophós $^{\circledR} \quad \mathrm{e}$ Metamidophós ${ }^{\circledR}$ ) e aplicações do fungo Sporothrix insectorum (Hoog \& Evans) incorporado na calda durante o período estudado.

As amostragens foram realizadas em árvores da bordadura do quadrante sul dos talhões estudados e distanciadas a aproximadamente $13 \mathrm{~m}$ das bordaduras do fragmento de Cerrado e da Mata ripária, sendo separados por uma estrada de terra. Os quadrantes norte, leste e oeste dos talhões estudados fazem bordadura com outros talhões de seringueira.

Foram realizadas oito amostragens quinzenais nos talhões estudados, em cinco árvores da bordadura em cada um dos talhões, obedecendo a um espaçamento mínimo de $20 \mathrm{~m}$ entre elas. 
Tabela 1. Características dos talhões de seringueira amostrados (clone PR 255) no período de outubro de 2005 a fevereiro de 2006, em Itiquira, MT.

\begin{tabular}{cccc}
\hline Talhão & Tamanho (ha) & Idade $($ anos $)$ & Altura média das árvores $(\mathrm{m})$ \\
\hline 610 B & 15,3 & 22 & 15 \\
712 B & 24,1 & 19 & 13 \\
\hline
\end{tabular}

Foram coletadas oito folhas maduras (completamente expandidas) por árvore, no terço inferior da copa, que apresentavam sintomas de ataque de $L$. heveae, totalizando 40 folhas por talhão/amostragem. Cividanes et al. (2004) verificaram que a distribuição de ninfas e adultos de $L$. heveae nas folhas de seringueira dá-se de maneira similar nos diferentes estratos da planta, não comprometendo a amostragem vertical.

As folhas foram acondicionadas em sacos de papel identificados e levadas ao laboratório para posterior avaliação. No laboratório foi escolhido aleatoriamente um folíolo por folha/árvore, sendo lavadas as superfícies abaxiais e adaxiais dos mesmos em solução de hipoclorito de sódio $(1,5 \%)$, com auxílio de um pincel macio para retirada de sujidades e ovos de outros insetos. Após, eram enxaguados em água destilada e mantidos em papel absorvente até a secagem.

Os pecíolos de cada folíolo foram inseridos em tubos plásticos contendo água destilada, tendo sua abertura lacrada com Parafilm $\mathbf{M}^{\circledR}$ para evitar o escoamento da mesma. Esse material (tubos + folíolos) foi acondicionado em sacos plásticos $(12 \mathrm{~cm} \mathrm{x}$ $30 \mathrm{~cm}$ ) identificados, inflados com um compressor de ar e fechados com auxílio de seladora elétrica. Os sacos foram transferidos para uma sala climatizada $(25 \pm$ $1{ }^{\circ} \mathrm{C}$, fotofase de 12 horas) e pendurados em varais, seguindo a metodologia de Santos \& Freitas (2008). Os ovos endofíticos de $L$. heveae não foram contabilizados nos ensaios.

Após cinco dias os sacos plásticos e os folíolos foram observados sob estereomicroscópio e contabilizados o número de parasitoides adultos emergidos.
Com auxílio de instrumento pontiagudo, os ovos de $L$. heveae foram dissecados a procura dos não emergidos.

Foi utilizado o teste de Qui-quadrado $(\alpha=5 \%$ ) para testar a associação entre $E$. tingitiphagus e o tipo de vegetação adjacente à bordadura dos talhões. A diferença na abundância de parasitoides em relação ao tipo de vegetação foi determinada através do teste $\mathrm{T}$ de Student $(\alpha=1 \%)$. A uniformidade ao longo das linhas de vegetação foi testada pelo teste não-paramétrico de KruskalWallis $(\alpha=1 \%)$.

Os parasitoides encontrados foram preservados em frascos de vidro contendo etanol $(80 \%)$ e identificados pelo Dr. Valmir Antonio Costa (Instituto Biológico/APTA, Campinas, SP).

\section{RESULTADOS E DISCUSSÃO}

Do total de 640 folíolos analisados foram encontrados 254 mimarídeos da espécie Erythmelus tingitiphagus sendo verificada uma ocorrência de 39,7\% (Tabela 2). Foram encontradas duas outras espécies de parasitoides, Schizophragma bicolor (Dozier) (Hymenoptera: Mymaridae) e Eretmocerus sp. (Hymenoptera: Aphelinidae) que foram desconsiderados nas análises deste estudo, por ocorrerem em baixa quantidade e por não terem relação associada a $L$. heveae.

Nos ensaios realizados foram encontradas ninfas de cicadelídeos nas amostras examinadas, portanto não se pode determinar com certeza a relação de parasitismo destas espécies com L. heveae, assim como relatado no trabalho de Santos \& Costa (2008). 
Tabela 2. Número total de Erythmelus tingitiphagus em folíolos de seringueira do clone PR 255, vizinhos a um fragmento de Cerrado e Mata ripária, no período de outubro de 2005 a fevereiro de 2006 em Itiquira, MT.

\begin{tabular}{ccc}
\hline Datas & Cerrado & Mata Ripária \\
\hline $20 / 10 / 05$ & 37 & 09 \\
$04 / 11 / 05$ & 14 & 07 \\
$19 / 11 / 05$ & 06 & 05 \\
$04 / 12 / 05$ & 68 & 26 \\
$19 / 12 / 05$ & 16 & 11 \\
$03 / 01 / 06$ & 10 & 29 \\
$18 / 01 / 06$ & 12 & 04 \\
$02 / 02 / 06$ & 00 & 00 \\
\hline TOTAL & 163 & 91 \\
\hline
\end{tabular}

O maior número de espécimes de $E$. tingitiphagus foi obtido em folíolos das árvores que fazem bordadura com Cerrado, apresentando uma média de 0,51 parasitoide/folíolo em relação à Mata ripária com 0,28 parasitoides/folíolo $(\mathrm{t}=2,83 ; \mathrm{gl}=$ $524,6 ; \mathrm{p}=0,004)$, representando uma abundância de quase o dobro no Cerrado. No entanto, este valor está abaixo do verificado por Santos \& Freitas (2008), com média de 1,7 parasitoide/folíolo, para o clone PR 255 em talhão policlonal.

$\mathrm{O}$ maior número de amostragens realizadas no estudo de Santos \& Freitas (2008), certamente contribuiu para o aumento da média de parasitoides/folíolo, além do fato das amostragens de folhas não terem sido realizadas somente na bordadura do talhão, diminuindo a influência do efeito de borda sobre a população do parasitoide.

A distribuição de E. tingitiphagus se mostrou uniforme ao longo da linha das árvores da bordadura do Cerrado $[\mathrm{H}(4,320)$ $=7,24 ; \mathrm{p}=0,1237]$ e quase uniforme na bordadura Mata ripária $[\mathrm{H}(4,320)=12,93 ; \mathrm{p}$ $=$ 0,0116]. Santos e Freitas (2008), estudando a distribuição horizontal e sazonal de E. tingitiphagus em plantios de seringueira, em Itiquira, MT, verificaram uma tendência do aumento do número de parasitoides da bordadura ao centro do talhão. Segundo Tabanez et al. (1997), o efeito de borda seria a influência do meio externo na porção marginal de áreas florestadas, causando alterações físicas e estruturais, como aumento nos níveis de luz, temperatura, umidade e vento (Kapos, 1989; Bierregaard et al. 1992; Rodrigues, 1998). Estes fatores podem provocar a migração de $L$. heveae da borda para o centro do talhão, em busca de melhores condições de sobrevivência e reprodução e, consequientemente, de E. tingitiphagus, pelo aumento da disponibilidade de hospedeiros em direção ao centro do talhão. Santos et al. (2002) relataram que a abundância de insetos herbívoros foi menor na vegetação nativa (Mata Atlântica e Cerrado) e em suas zonas de contato com plantios de eucalipto do que no centro destes.

Foi verificado que as diferenças na ocorrência de E. tingitiphagus nos dois talhões $(64,2 \%$ no Cerrado e $35,8 \%$ na Mata ripária) não foram devido ao acaso $\left(\chi^{2}=\right.$ 5,016; $\mathrm{gl}=1 ; \mathrm{p}=0,025)$, evidenciando uma preferência desta espécie pelo Cerrado. Foi verificada uma maior abundância e diversidade de fanerógamas no Cerrado em relação à Mata ripária, oque possivelmente seja um dos fatores que influenciaram positivamente na ocorrência de $E$. tingitiphagus neste bioma. $\mathrm{O}$ néctar das flores configura um suprimento alimentar extra para os adultos do parasitoide, além da 
possibilidade de hospedeiros alternativos e refúgio nesta área (Vinson \& Barbosa, 1987; Altieri, 1994). Foi observado um menor número de plantas com flores na Mata ripária, principalmente devido à ação antrópica na área, e conseqüentemente houve diminuição dos recursos alimentares e de micro-habitats favoráveis para os parasitoides, determinando um menor nível populacional nesta área.

O número máximo de indivíduos coletados de E. tingitiphagus ocorreu no mês de dezembro de 2005 em ambos os talhões. Este resultado concorda com o obtido por Santos \& Freitas (2008) que relataram picos populacionais de $L$. heveae e $E$. tingitiphagus nos meses de novembro e dezembro nesta região. A ausência de parasitoides obtidos nas coletas de fevereiro de 2006 provavelmente esteja mais relacionada ao declínio populacional de $L$. heveae na área, conforme relatado em Santos \& Freitas (2008), do que propriamente relacionada ao efeito de borda.

Erythmelus tingitiphagus ocorreu na bordadura dos talhões estudados, no entanto, apresentando um nível populacional abaixo dos verificados por Santos \& Freitas (2008) e Santos et al. (2009). Possivelmente, um dos fatores que influenciaram nesta redução populacional foi o efeito de borda, proporcionando condições desfavoráveis tanto à praga quanto ao seu inimigo natural, como aumento de temperatura, insolação, vento, diminuição de umidade, entre outros, associado à menor quantidade de hospedeiros (ovos) disponíveis nas árvores da bordadura. Todavia, a preservação de vegetação de entorno (principalmente com grande número de fanerógamas) adjacente aos talhões de seringueira, pode proporcionar condições favoráveis à permanência de parasitoide nos mesmos. Estas áreas de maior diversidade podem servir de habitat para várias espécies de predadores e parasitoides, promovendo um controle biológico natural e preservando remanescentes de vegetação nativa.

\section{CONCLUSÃO}

O efeito de borda atua negativamente na população de Erythmelus tingitiphagus, diminuindo sua população nesta faixa.

O fragmento de Cerrado atuou positivamente no aumento populacional de Erythmelus tingitiphagus em árvores da bordadura, em relação ao vizinho à Mata Ripária.

\section{AGRADECIMENTOS}

Aos funcionários Braz da Silva e Silmar Dias Ferreira, pela ajuda na condução dos experimentos. A Luciana Maira de Sales Pereira pela revisão do Abstract. À empresa "Plantações Edouard Michelin Ltda." pelo financiamento do projeto e apoio logístico para a realização do estudo.

\section{REFERÊNCIAS BIBLIOGRÁFICAS}

ALTIERI, M.A. 1994. Biodiversity and pest management in agroecosystems. New York: Haworth Press. 185p.

ALTIERI, M.A.; SILVA, E.N.; NICHOLLS, C.I. 2003. O papel da biodiversidade no manejo de pragas. Ribeirão Preto: Holus. 226p.

BIERREGAARD, R.O.; LOVEJOY, T.E.; KAPOS, V.; SANTOS, A.A.; HUTCHINGS, R.W. 1992. The biological dynamics of tropical rainforest fragments. Bioscience, v. 42, p.859-866.

BORROR, J.D.; DeLONG, D.M. 1988. Introdução ao estudo dos insetos. São Paulo: Edgard Blucher. 653p.

CADENASSO, M.L.; PICKETT, S.T.A. 2000. Linking Forest edge structure and to edge function: mediation of herbivore damage. Journal of Ecology, v. 88, p. 31-44. 
CIVIDANES, F.J.; FONSECA, F. S.; SANTOS, T. M. 2004. Distribuição de Leptopharsa heveae em seringal no Estado de São Paulo. Pesquisa Agropecuária Brasileira, v. 39, n. 10, p. 1053-1056.

COSTA, V.A.; PEREIRA, C. de F.; BATISTA FILHO, A. 2003. Observações preliminares sobre o parasitismo de ovos de Leptopharsa heveae (Hemiptera: Tingidae) em seringueira, em Pindorama, SP. Arquivos do Instituto Biológico, v. 70, n. 2, p. 205-206.

GALLO, D.; NAKANO, O.; SILVEIRA NETO, S.; CARVALHO, R.P.L.; BAPTISTA, G.C.; BERTI FILHO, E.; PARRA, J.R.P.; ZUCCHI, R.A.; VENDRAMIM, J.D.; MARCHINI, L.C.; LOPES, J.R.S.; OMOTO, C. 2002. Entomologia Agrícola. Piracicaba: FEALQ. 920p.

GIRÃO, L.C.; LOPES, A.V.; TABARELLI, M.; BRUNA, E. 2007. Changes in tree reproductive traits reduce functional diversity in a fragmented Atlantic Forest landscape. Plos one. v. 9, p. 1-12.

KAPOS, V. 1989. Effects of isolation on the water status of Forest patches in the Brazilian Amazon. Journal of Tropical Ecology, v. 5, p. 173-185.

LASALLE, J.; GAULD, I.D. 1993. Hymenoptera and biodiversity. Wallingford, UK: C.A.B. International. $348 \mathrm{p}$.

MARCHIORI, C.H.; SILVA, C.G.; CALDAS, E.R.; ALMEIDA, K.G.S.; CARVALHO, S.A.; PENTEADO-DIAS, A.M.; DIAZ, N.B.; GALLARDO, F.E. 2001. Parasitoides da subfamília Eucoilinae (Hymenoptera: Cynipoidea: Figitidae) coletados em um remanescente de mata de cerrado em Itumbiara, GO. Arquivos do Instituto Biológico, v. 68, n. 1, p. 65-67.

MURCIA, C. 1985. Edge effect in fragmented forests: implications for conservation. Tree. v. 10, n. 7, p. 759763.

RODRIGUES, E. 1998. Edge effects on the regeneration of forest fragments in south Brazil. Tese de doutorado. Harvard University, Cambridge, Massachusetts. $172 p$.

SANTOS, G.P.; ZANUNCIO, T.V.; VINHA, E.; ZANUNCIO, J.C. 2002. Influência de faixas de vegetação nativa em povoamentos de Eucalyptus cloeziana sobre a população de Oxydia vesulia (Lepidoptera: Geometridae). Revista Árvore, v. 26, p. 459-504.

SANTOS, R.S.; COSTA, V.A. 2008. Primeiro registro de Schizophragma bicolor (Dozier, 1932) (Hymenoptera: Mymaridae) no Brasil. Revista de Agricultura, v. 83, n. 2, p. 140-143.

SANTOS, R.S.; FREITAS, S. de. 2008. Parasitismo de Erythmelus tingitiphagus (Soares) (Hymenoptera: Mymaridae) em ovos de Leptopharsa heveae Drake \& Poor (Hemiptera: Tingidae), em plantios de seringueira (Hevea brasiliensis Müell. Arg.). Neotropical Entomology, v. 37, n. 5, p. 571-576.

SANTOS, R.S.; FREITAS, S. de; SILVA, J. M. 2009. Distribuição horizontal sazonal de Erythmelus tingitiphagus em plantios de seringueira. Revista de Ciências Agroveterinárias, v. 8, n. 1, p. 53-61.

SILVA, P.S.D.; LEAL, I.R.; WIRTH, R.; TABARELLI, M. 2007. Harvesting of Protium heptaphyllum Aublet (March.) seeds (Burseraceae) by the leaf-cutting ant Atta sexdens L. promotes seed aggregation and seedling mortality. Revista Brasileira de Botânica, v. 30, p. 553-560.

TABANEZ, A.A.J.; VIANA, V.M.; DIAS, A.S. 1997. Conseqüências da fragmentação e do efeito de borda sobre a estrutura, diversidade e sustentabilidade de um fragmento de floresta de planalto de Piracicaba, SP. Revista Brasileira de Biologia, v. 57, p. 47-60. 
VINSON, S.B.; BARBOSA, P. 1987.

Interrelationship of nutritional ecology of parasitoids. In: SLANSKY JR., F.; RODRIGUES, J.G. (eds.). Nutritional ecology of insects, mites, spiders and related invertebrates. New York: John Wiley \& Sons. 1016p. 